\title{
Pacific
}

Journal of

Mathematics

\section{NORMAL STATES OF TYPE III FACTORS}

YASUYUKI KaWAHIGASHI, Yoshiko Ogata AND ERLING STøRMER 


\title{
NORMAL STATES OF TYPE III FACTORS
}

\author{
Yasuyuki KaWAhigashi, Yoshiko OGata AND ERLING Størmer
}

Dedicated to Masamichi Takesaki on the occasion of his eightieth birthday.

\begin{abstract}
Let $M$ be a factor of type III with separable predual and with normal states $\varphi_{1}, \ldots, \varphi_{k}, \omega$ with $\omega$ faithful. Let $A$ be a finite-dimensional $C^{*}$-subalgebra of $M$. Then it is shown that there is a unitary operator $u \in M$ such that $\varphi_{i} \circ \operatorname{Ad} u=\omega$ on $A$ for $i=1, \ldots, k$. This follows from an embedding result of a finite-dimensional $C^{*}$-algebra with a faithful state into $M$ with finitely many given states. We also give similar embedding results of $C^{*}$-algebras and von Neumann algebras with faithful states into $M$. Another similar result for a factor of type $\mathrm{II}_{1}$ instead of type III holds.
\end{abstract}

\section{Introduction}

Let $M$ be a factor of type III with separable predual. Then two nonzero projections $e$ and $f$ in $M$ are equivalent, that is, there exists a partial isometry $v \in M$ such that $v^{*} v=e, v v^{*}=f$. If, furthermore, $e$ and $f$ are different from the identity operator 1 , then there is a unitary operator $u \in M$ such that $u^{*} e u=f$. This shows that there is an abundance of unitaries in $M$, so one might expect stronger results arising from these unitaries. That is what is done in the present paper. We show that if $\varphi$ and $\omega$ are faithful normal states in $M$ and $A \subset M$ is a finite-dimensional $C^{*}$-algebra, then there exists a unitary operator $u \in M$ such that the restrictions $\left.\varphi \circ \operatorname{Ad} u\right|_{A}$ and $\left.\omega\right|_{A}$ are equal, where $\operatorname{Ad} u$ is the inner automorphism $x \mapsto u^{*} x u$ of $M$. (See Corollary 2.2 for a more precise and general statement.)

This actually follows from an embedding result of a finite-dimensional $C^{*}$ algebra $A$ with a faithful state into $M$ with finitely given normal states. This result is then applied to obtain a similar result for the $C^{*}$-algebra of the compact operators on a separable Hilbert space. Furthermore, we have more general embedding results in Section 3 for $C^{*}$-algebras and von Neumann algebras with faithful states into a

Kawahigashi is supported in part by the Global COE Program "The research and training center for new development in mathematics", the Mitsubishi Foundation Research Grants and the Grantsin-Aid for Scientific Research, JSPS. Ogata is supported in part by the Inoue Foundation and the Grants-in-Aid for Scientific Research, JSPS.

MSC2010: 46L30.

Keywords: von Neumann algebra, type III factor, normal state. 
type III factor $M$ such that a finite number of normal states on $M$ coincide after the embedding.

If $M$ is not of type III, the corresponding result is false in general, but if $M$ is a factor of $\mathrm{II}_{1}, \omega=\tau$ is the trace and $A \cong M_{n}(\mathbb{C})$, the matrix algebra of complex $n \times n$-matrices, then the corresponding result to the unitary equivalence on $A$ holds for $\omega=\tau$ and any $\varphi$. This will be shown in Section 4 .

There exist results of a similar nature to the ones above in the literature. In [Connes and Størmer 1978], it has been shown that if $M$ is of type $\mathrm{III}_{1}$ and $\varepsilon>0$ then there is a unitary operator $u \in M$ with

$$
\|\varphi \circ \operatorname{Ad} u-\omega\|<\varepsilon .
$$

If one takes a pointwise weak limit point of the automorphisms of the form $\operatorname{Ad} u$ in the above, then one finds a completely positive unital map $\pi: M \rightarrow M$ with $\varphi \circ \pi=\omega$.

In the nonseparable case, it has recently been shown by Ando and Haagerup [2013] that for some factors of type $\mathrm{III}_{1}$ constructed as ultraproducts, all faithful normal states are unitarily equivalent.

In the $C^{*}$-algebra case it has been shown in [Kishimoto et al. 2003] that if $\varphi$ and $\omega$ are pure states of a separable $C^{*}$-algebra $A$ with the same kernel for their GNS-representations, then there is an asymptotically inner automorphism $\alpha$ of $A$ such that $\varphi \circ \alpha=\omega$.

Our result gives an exact equality for two states, not an approximate one, but only on a finite-dimensional $C^{*}$-subalgebra $A$.

\section{Factors of type III}

In this section we state and prove our main result.

Theorem 2.1. Let $M$ be a type III factor with separable predual and $\varphi_{1}, \ldots, \varphi_{k}$ normal states on $M$. Let $A$ be a finite-dimensional $C^{*}$-algebra and $\rho$ a faithful state on A. Then there exists a unital injective homomorphism $\pi: A \rightarrow M$ with

$$
\varphi_{i} \circ \pi=\rho, \quad i=1, \ldots, k .
$$

After proving this theorem, we will prove that it implies the following corollary.

Corollary 2.2. Let $M$ be a factor of type III with separable predual. Let $A$ be a finite-dimensional $C^{*}$-subalgebra of $M$. Let $\varphi_{1}, \ldots, \varphi_{k}$ and $\omega$ be normal states on $M$ and assume that $\omega$ is faithful. Then there exists a unitary operator $u \in M$ such that

$$
\left.\varphi_{i} \circ \operatorname{Ad} u\right|_{A}=\left.\omega\right|_{A}, \quad i=1, \ldots, k .
$$


Before starting preliminaries of our proof of Theorem 2.1, we give an outline of our method for the case $A \cong M_{d}(\mathbb{C})$.

After diagonalizing the density matrix of $\rho$, what we have to find is a system of matrix units $\left\{e_{i j}\right\}$ in $M$ for which we have $\varphi_{n}\left(e_{i j}\right)=\delta_{i j} \lambda_{i}$ for all $n=1, \ldots, k$ and $i, j=1, \ldots, d$, where the $\lambda_{i}$ are eigenvalues of the density matrix of $\rho$. We first choose $e_{i i}$ satisfying this condition. Then we choose $e_{12}, e_{13}, \ldots, e_{1 d}$ inductively so that we have various identities saying that the values of certain linear functionals applied to a certain partial isometry are all zero at each induction step. This is done by a version of a noncommutative Lyapunov theorem, and what we need is a special case of [Akemann and Anderson 1991, Theorem 2.5(1)]. Since the statement and its proof are short, we include them here in the form we need, for the sake of convenience of the reader.

Lemma 2.3. Let $M$ be a nonatomic von Neumann algebra and $\Phi: M \rightarrow \mathbb{C}^{n}$ a $\sigma$-weakly continuous linear map. Then for any $a \in M_{+, 1}$, there exists a projection $p \in M$ such that $\Phi(p)=\Phi(a)$.

Proof. Let

$$
D:=\left\{x \in M_{+, 1} \mid \Phi(x)=\Phi(a)\right\},
$$

where $M_{+, 1}$ denotes the positive operators in the unit ball of $M$. Then $D$ is a nonempty $\sigma$-weakly compact convex set. Therefore, by the Krein-Milman theorem, there exists an extremal point $b$ of $D$. We show $b$ is a projection. If $b$ were not a projection, then there exists $\delta \in\left(0, \frac{1}{2}\right)$ such that the spectral projection $p$ of $b$ corresponding to $(\delta, 1-\delta)$ is nonzero. By the assumption on $M, p M_{\mathrm{sa}} p$ is an infinite-dimensional real linear space while its range with respect to $\Phi$ is finitedimensional. This implies the existence of a nonzero $y \in p M_{\mathrm{sa}} p$ such that $\Phi(y)=0$. Setting $t:=\delta /\|y\|$, we have $b \pm t y \in D$. As we have $b=(b+t y) / 2+(b-t y) / 2$, this contradicts the fact that $b$ is extremal in $D$.

We now construct appropriate matrix units by induction on the size of matrix units.

Lemma 2.4. Let $M$ be a type III factor with separable predual and $\varphi_{1}, \ldots, \varphi_{n}$ normal states on $M$. Let $\lambda_{i}>0, i=1, \ldots, m$ with $\sum_{i} \lambda_{i}=1$. Then there exists $a$ system of matrix units $\left\{e_{i j}\right\}_{i, j=1, \ldots, m}$ such that

$$
\varphi_{l}\left(e_{i j}\right)=\delta_{i j} \lambda_{i} \quad \text { for all } l=1, \ldots, m .
$$

Proof. For a projection $p \in M$ satisfying $0 \leq \varphi_{l}(p)=\lambda<1$ for $l=1, \ldots, n$ and $0 \leq t \leq 1-\lambda$, there exists a projection $q$ orthogonal to $p$ such that $\varphi_{l}(q)=t$. To see this, we consider a $\sigma$-weakly continuous liner map $\Phi: M_{\bar{p}} \rightarrow \mathbb{C}^{n}$, where we write $\bar{p}=1-p$, given by $\Phi(x)=\left(\varphi_{l}(x)\right)_{l=1}^{n}$, and apply Lemma 2.3 for $a=t \bar{p} /(1-\lambda)$.

Using this fact inductively, we have $\left\{e_{i i}\right\}$. 
We next define partial isometries $u_{i 1}, i=1, \ldots, m$, inductively such that $e_{i j}=$ $u_{i 1} u_{j 1}^{*}$ satisfy the conditions of the lemma. Let $u_{11}=e_{11}$ and assume that we have found $u_{i 1}, i=1, \ldots, k$ with $k<m$. Let $v$ be a partial isometry in $M$ with $v^{*} v=e_{11}$, $v v^{*}=e_{k+1, k+1}$. Then define a map

$$
\begin{gathered}
\Phi: e_{11} M e_{11} \rightarrow \mathbb{C}^{n k} \\
\Phi(x):=\left(\varphi_{l}\left(v x u_{j 1}^{*}\right)\right)_{l=1, \ldots, n, j=1, \ldots, k} .
\end{gathered}
$$

This map $\Phi$ is $\sigma$-weakly continuous and linear, so by using Lemma 2.3 with $a=e_{11} / 2$, we obtain a projection $p \in e_{11} M e_{11}$ such that $\Phi(p)=\Phi\left(e_{11}\right) / 2$. Define

$$
u_{k+1,1}:=v p-v(1-p) .
$$

Since $p \leq e_{11}$, an easy computation shows that $u_{k+1,1}^{*} u_{k+1,1}=e_{11}, u_{k+1,1} u_{k+1,1}^{*}=$ $e_{k+1, k+1}$. Let $e_{k+1, j}=u_{k+1,1} u_{j 1}^{*}$ and $e_{j, k+1}=u_{j 1} u_{k+1,1}^{*}$. Then the $e_{i j}, i, j \leq k+1$, form a set of matrix units, and using the definition of $\Phi$ and that $\Phi(p)=\Phi\left(e_{11}\right) / 2$, we get for all $l$

$$
\begin{aligned}
\varphi_{l}\left(u_{k+1,1} u_{j 1}^{*}\right) & =\varphi_{l}\left((2 v p-v) u_{j 1}^{*}\right) \\
& =2 \varphi_{l}\left(v p u_{j 1}^{*}\right)-\varphi_{l}\left(v u_{j 1}^{*}\right) \\
& =0 .
\end{aligned}
$$

Thus

$$
\varphi_{l}\left(e_{j, k+1}\right)=\varphi_{l}\left(u_{j 1} u_{k+1,1}^{*}\right)=\overline{\varphi_{l}\left(u_{k+1,1} u_{j 1}^{*}\right)}=0,
$$

completing the proof of the lemma.

Proof of Theorem 2.1. First we consider the case $A=M_{m}(\mathbb{C})$. We choose a system of matrix units $\left\{v_{i j}\right\}_{i, j=1, \ldots, m}$ of $A=M_{m}(\mathbb{C})$ which diagonalizes the density matrix $D_{\rho}$ of $\rho$, that is, $D_{\rho}=\sum_{i=1}^{m} \lambda_{i} v_{i i}$. As $\rho$ is faithful, we have $\lambda_{i}>0$ for all $i$. By Lemma 2.4, we obtain a system of matrix units $\left\{e_{i j}\right\}_{i, j=1, \ldots, m}$ in $M$ satisfying

$$
\varphi_{n}\left(e_{i j}\right)=\delta_{i j} \lambda_{i}, \quad n=1, \ldots, k, \quad i, j=1, \ldots, m .
$$

Define

$$
\pi: M_{m}(\mathbb{C}) \rightarrow M, \quad \pi\left(v_{i j}\right)=e_{i j} .
$$

Then $\pi$ gives a unital homomorphism satisfying the desired condition.

For the general case $A \simeq \bigoplus_{k=1}^{b} M_{n_{k}}(\mathbb{C})$, let $m=\sum_{k=1}^{b} n_{k}$. Let $\hat{\rho}$ be a faithful extension of $\rho$ to $M_{m}(\mathbb{C})$. Applying the above result to $M_{m}(\mathbb{C})$ and $\hat{\rho}$, there exists a unital homomorphism $\hat{\pi}: M_{m}(\mathbb{C}) \rightarrow M$ such that

$$
\varphi_{n} \circ \hat{\pi}=\hat{\rho}, \quad n=1, \ldots, k .
$$

The restriction $\pi:=\left.\hat{\pi}\right|_{A}$ gives a unital homomorphism from $A$ to $M$ satisfying $\varphi_{n} \circ \pi=\rho$, for $n=1, \ldots, k$. 
Proof of Corollary 2.2. Let $p$ be the unit of $A$. Considering $A \oplus \mathbb{C}(1-p)$ instead of $A$, we may assume that $A$ contains the unit of $M$ from the beginning.

First we consider the case $A \simeq M_{m}(\mathbb{C}), m \in \mathbb{N}$. Let $\left\{f_{i j}\right\}_{i, j=1, \ldots, m},\left\{v_{i j}\right\}_{i, j=1, \ldots, m}$ be systems of matrix units of $A$ and $M_{m}(\mathbb{C})$, respectively. Let $\gamma: M_{m}(\mathbb{C}) \rightarrow A$ be an isomorphism given by $\gamma\left(v_{i j}\right)=f_{i j}$.

Then $\rho:=\omega \circ \gamma$ is a faithful state on $M_{m}(\mathbb{C})$. From Theorem 2.1, there exists a unital homomorphism $\pi: M_{m}(\mathbb{C}) \rightarrow M$ such that $\varphi_{n} \circ \pi=\rho, n=1, \ldots, k$. The algebras $A$ and $\pi\left(M_{m}(\mathbb{C})\right)$ are subalgebras of $M$ isomorphic to $M_{m}(\mathbb{C})$ with complete sets of matrix units $\left\{f_{i j}\right\}$ and $\left\{\pi\left(v_{i j}\right)\right\}$. As in [Haagerup and Musat 2011, Lemma 2.1], if $v \in M$ is a partial isometry with $v^{*} v=\pi\left(v_{11}\right)$ and $v v^{*}=f_{11}$, then $u:=\sum_{i=1}^{m} \pi\left(v_{i 1}\right) v^{*} f_{1 i}$ is a unitary in $M$ satisfying $u f_{i j} u^{*}=\pi\left(v_{i j}\right)$. Hence we have

$$
\varphi_{n} \circ \operatorname{Ad} u\left(f_{i j}\right)=\varphi_{n}\left(\pi\left(v_{i j}\right)\right)=\rho\left(v_{i j}\right)=\omega \circ \gamma\left(v_{i j}\right)=\omega\left(f_{i j}\right),
$$

that is, $\left.\varphi_{n} \circ \operatorname{Ad} u\right|_{A}=\left.\omega\right|_{A}$ for $n=1, \ldots, k$.

For the general case $A \simeq \bigoplus_{l=1}^{b} M_{n_{l}}(\mathbb{C})$, let $\left\{f_{i j}^{(l)}\right\}_{i j=1, \ldots, n_{l}}$ be a system of matrix units of $M_{n_{l}}(\mathbb{C})$ for each $l=1, \ldots, b$. As $M$ is of type III, for all $l=1, \ldots, b$, the nonzero projections $f_{11}^{(1)}$ and $f_{11}^{(l)}$ are mutually equivalent. Hence, there exist partial isometries $v^{(l)} \in M$ such that $v^{(l)^{*}} v^{(l)}=f_{11}^{(l)}$ and $v^{(l)} v^{(l)^{*}}=f_{11}^{(1)}$. Set $w_{(k, i)(l, j)}:=f_{i 1}^{(k)} v^{(k)^{*}} v^{(l)} f_{1 j}^{(l)}$, for $k, l=1, \ldots, b, i=1, \ldots, n_{k}$, and $j=1, \ldots, n_{l}$. Then we have

$$
\begin{aligned}
w_{(k, i)(l, j)}^{*} & =f_{j 1}^{(l)} v^{(l)^{*}} v^{(k)} f_{1 i}^{(k)}=w_{(l, j)(k, i)}, \\
w_{(k, i)(l, j)} w_{\left(l^{\prime}, j^{\prime}\right)\left(k^{\prime}, i^{\prime}\right)} & =f_{i 1}^{(k)} v^{(k)^{*}} v^{(l)} f_{1 j}^{(l)} f_{j^{\prime} 1}^{\left(l^{\prime}\right)} v^{\left(l^{\prime}\right)^{*}} v^{\left(k^{\prime}\right)} f_{1 i^{\prime}}^{\left(k^{\prime}\right)} \\
& =\delta_{l l^{\prime}} \delta_{j j^{\prime}} f_{i 1}^{(k)} v^{(k)^{*}} v^{(l)} f_{11}^{(l)} v^{(l)^{*}} v^{\left(k^{\prime}\right)} f_{1 i^{\prime}}^{\left(k^{\prime}\right)} \\
& =\delta_{l l^{\prime}} \delta_{j j^{\prime}} f_{i 1}^{(k)} v^{(k)^{*}} v^{(l)} v^{(l)^{*}} v^{\left(k^{\prime}\right)} f_{1 i^{\prime}}^{\left(k^{\prime}\right)} \\
& =\delta_{l l^{\prime}} \delta_{j j^{\prime}} w_{(k i),\left(k^{\prime} i^{\prime}\right)}, \\
\sum_{(k, i)} w_{(k, i)(k, i)} & =\sum_{i, k} f_{i 1}^{(k)} v^{(k)^{*}} v^{(k)} f_{1 i}^{(k)}=\sum_{(k, i)} f_{i i}^{(k)}=1 .
\end{aligned}
$$

Hence $\left\{w_{(k, i)(l, j)}\right\}_{(k, i),(l, j)}$ give a system of matrix units of a $C^{*}$-subalgebra $B$ of $M$ isomorphic to $M_{m}$, for $m:=\sum_{k=1}^{b} n_{k}$. As $w_{(k i)(k j)}=f_{i 1}^{(k)} f_{1 j}^{(k)}=f_{i j}^{(k)}$, $\left\{w_{(k, i)(l, j)}\right\}$ is an extension of $\left\{f_{i j}^{(k)}\right\}$ and $A$ is a subalgebra of $B$. We apply the above argument to $B \simeq M_{m}(\mathbb{C})$ and obtain a unitary $u$ in $M$ such that $\left.\varphi_{i} \circ \operatorname{Ad} u\right|_{B}=\left.\omega\right|_{B}$. In particular, we obtain $\left.\varphi_{i} \circ \operatorname{Ad} u\right|_{A}=\left.\omega\right|_{A}$ for $i=1, \ldots, k$.

\section{Embedding of operator algebras with faithful states}

The above theorem can be extended to the algebra of the compact operators as follows. 
Theorem 3.1. Let $K(\mathscr{H})$ denote the set of all the compact operators on a separable Hilbert space Het Let $\rho$ be a faithful state on $K(\mathscr{H})$. Let $M$ be a factor of type III with separable predual, $\varphi_{1}, \varphi_{2}, \ldots, \varphi_{k}$ normal states on $M$. Then there exists a homomorphism $\pi$ of $K(\mathscr{H})$ into $M$ such that

$$
\varphi_{n} \circ \pi=\rho, \quad n=1, \ldots, k .
$$

Proof. We may assume that $\mathscr{H}$ is infinite-dimensional and $\varphi_{1}$ is faithful - for example, by adding a faithful state to the set of all the $\varphi_{i}$.

Let $\left\{v_{i j}\right\}$ be a system of matrix units of $K(\mathscr{H})$ diagonalizing the density matrix $D_{\rho}$ of $\rho$, that is, $D_{\rho}=\sum_{i=1}^{\infty} \lambda_{i} v_{i i}$. As $\rho$ is faithful, we have $\lambda_{i}>0$ for all $i$.

We claim that there exists a system of matrix units $\left\{e_{i j}\right\}_{i, j \in \mathbb{N}}$ in $M$ satisfying

$$
\varphi_{n}\left(e_{i j}\right)=\delta_{i j} \lambda_{i}, \quad n=1, \ldots, k, \quad i, j=1,2, \ldots
$$

This is proved in the same way as in the proof of Theorem 2.1.

A slight rewriting of the above theorem gives the following:

Corollary 3.2. Let $B(\mathscr{H})$ be the set of all the bounded operators on a separable Hilbert space $\mathscr{H}$ and $\rho$ a faithful normal state on $B(\mathscr{H})$. Let $M$ be a factor of type III with separable predual and $\varphi_{1}, \varphi_{2}, \ldots, \varphi_{k}$ normal states on $M$. Then there exists a homomorphism $\pi$ of $B(\mathscr{H})$ into $M$ such that

$$
\varphi_{n} \circ \pi=\rho, \quad n=1, \ldots, k .
$$

We now consider an embedding of a $C^{*}$-algebra with a faithful state into a type III factor with finitely many normal states.

Theorem 3.3. For a $C^{*}$-algebra $A$ and a faithful state $\omega$ on $A$, the following conditions are equivalent:

(i) The Hilbert space $\mathscr{H}_{\omega}$ in the GNS triple $\left(\mathscr{H}_{\omega}, \pi_{\omega}, \Omega_{\omega}\right)$ of $\omega$ is separable and $\Omega_{\omega}$ is separating for $\pi_{\omega}(A)^{\prime \prime}$.

(ii) There exists a representation (H, $\rho)$ of $A$ on a separable Hilbert space $\mathscr{H}$ and a faithful normal state $\sigma$ on $B(\mathscr{H})$ with $\omega=\sigma \circ \rho$.

(iii) For any factor $M$ of type III with separable predual and its normal states $\varphi_{1}, \ldots, \varphi_{n}$, there exists an injective homomorphism $\gamma: A \rightarrow M$ with $\varphi_{j} \circ \gamma=\omega$ for all $j=1, \ldots, n$.

Proof. Suppose condition (i) holds. Then $\Omega_{\omega}$ is cyclic for $\pi_{\omega}(A)^{\prime}$. Therefore, using the separability of $\mathscr{H}_{\omega}$, we have a sequence $\left\{x_{n}\right\}_{n=1}^{\infty} \subset\left(\pi_{\omega}(A)^{\prime}\right)_{1}$ such that $\left\{x_{n} \Omega_{\omega}: n \in \mathbb{N}\right\}$ spans $\mathscr{H}_{\omega}$. Let $x_{0}:=\sqrt{1-\sum_{n} x_{n}^{*} x_{n} / 2^{n}}$, and define a state $\sigma$ on $B\left(\mathscr{H}_{\omega}\right)$ given by the density matrix $\sum_{n=0}^{\infty}\left|x_{n} \Omega_{\omega}\right\rangle\left\langle x_{n} \Omega_{\omega}\right| / 2^{n}$. This $\sigma$ is faithful and normal. Let $\rho=\pi_{\omega}$. We can check $\sigma \circ \rho=\omega$. Hence (ii) holds. 
Now suppose condition (ii) holds. We show (iii). By Theorem 3.1, we have an injective homomorphism $\pi: K(\mathscr{H}) \rightarrow M$ such that $\left.\sigma\right|_{K(\mathscr{H})}=\varphi \circ \pi$. We denote the extension of $\pi$ to $B(\mathscr{H})$ by $\hat{\pi}$. Then from the way we have constructed $\pi$, we obtain $\sigma=\varphi \circ \hat{\pi}$. Define $\gamma:=\hat{\pi} \circ \rho: A \rightarrow M$. Then we obtain $\varphi \circ \gamma=\varphi \circ \hat{\pi} \circ \rho=\sigma \circ \rho=\omega$.

Finally suppose condition (iii) holds, and we show this implies (i). To see this, fix a factor $M$ of type III with a faithful normal state $\varphi$, and let $\left(\mathscr{H}_{\varphi}, \pi_{\varphi}, \Omega_{\varphi}\right)$ be its GNS triple. We obtain $\gamma$ as in (iii). Let $K:=\overline{\pi_{\varphi} \circ \gamma(A) \Omega_{\varphi}}$ and let $\beta$ be the restriction of $\pi_{\varphi} \circ \gamma$ to $K$. Then $\left(K, \beta, \Omega_{\varphi}\right)$ is the GNS triple of $\omega$. As $\Omega_{\varphi}$ is separating for $\pi_{\varphi}(M)$, it is separating for $\beta(A)^{\prime \prime}$, and (i) holds.

As an immediate corollary, we obtain the following:

Corollary 3.4. Let $N$ be a von Neumann algebra with separable predual and $\psi$ a faithful normal state on $N$. Then for any factor $M$ of type III with separable predual and a normal state $\varphi$ on $M$, there exists an injective homomorphism $\pi: N \rightarrow M$ with $\varphi \circ \pi=\psi$.

Another easy corollary is as follows, by a well-known result on the KMS condition [Bratteli and Robinson 1997, Corollary 5.3.9].

Corollary 3.5. Suppose that we have a $C^{*}$-algebra A, a state $\varphi$ on $A$, and a oneparameter automorphism group $\left\{\alpha_{t}\right\}_{t \in \mathbb{R}}$ such that these satisfy the KMS condition. Then the pair $(A, \varphi)$ satisfies the (equivalent) conditions in Theorem 3.3.

Remark 3.6. Note that a general faithful state on a $C^{*}$-algebra $A$ does not satisfy condition (i) of Theorem 3.3 at all, as shown in [Takesaki 1974] by an example due to Pedersen. The $C^{*}$-algebra used by Takesaki is a very basic one, $C([0,1]) \otimes M_{2}(\mathbb{C})$. A slight modification of the argument there also works for a simple $C^{*}$-algebra $A_{\theta} \otimes M_{2}(\mathbb{C})$, where $A_{\theta}$ is the irrational rotation $C^{*}$-algebra.

In Theorem 3 of the same paper, Takesaki gives a sufficient condition for our condition (i) in Theorem 3.3 and calls it the quasi-KMS condition, but it seems difficult to check this condition for a given example.

Remark 3.7. In all the above cases, we considered embeddings into a type III factor, but actually any properly infinite von Neumann algebra with separable predual works. This is because if we have a properly infinite von Neumann algebra and normal states on it, we simply restrict the states on a type III factor which is found as a subalgebra of the original von Neumann algebra. It is easy to see that if a von Neumann algebra with separable predual has a finite direct summand, this type of embedding is impossible, so actually this embeddability characterize proper infiniteness of a von Neumann algebra with separable predual. 


\section{Factors of type $\mathrm{II}_{1}$}

The direct analogue of Theorem 2.1 for finite factors is trivially false. For example, if $M$ is of type $\mathrm{II}_{1}$ with trace $\tau$ and $\rho$ is not a trace on $A$, then the conclusion of Theorem 2.1 for $\varphi_{1}=\tau$ is clearly false. However, if we restrict the choice of $\omega$ in Corollary 2.2, we obtain a positive result.

Theorem 4.1. Let $\varphi_{1}, \ldots, \varphi_{k}$ be normal states on a factor $M$ of type $I I_{1}$ with the unique trace $\tau$. Let $A$ be a $C^{*}$-subalgebra of $M$ isomorphic to $M_{m}(\mathbb{C})$ with $1 \in A$. Then there exists a unitary operator $u \in M$ satisfying $\left.\varphi_{i} \circ \operatorname{Ad} u\right|_{A}=\left.\tau\right|_{A}$ for $i=1, \ldots, k$.

Proof. We may assume that $\varphi_{1}=\tau$ is the unique trace on $M$. We proceed as in the proof of Theorem 2.1. The only difference is that we take $\tau\left(e_{i i}\right)=1 / m$ instead of the proof of Lemma 2.4 .

\section{Acknowledgements}

Y.K. thanks the CMTP in Rome and Université Paris VII, and Y.O. thanks University of Oregon and University of Oslo for hospitality during their stays when parts of this work were done. Y.K. is grateful to R. Longo and G. Skandalis for useful remarks, Y.O. to N. Christopher Phillips for helpful discussions and E.S. to S. Neshveyev.

\section{References}

[Akemann and Anderson 1991] C. A. Akemann and J. Anderson, Lyapunov theorems for operator algebras, Mem. Amer. Math. Soc. 458, Amer. Math. Soc., Providence, RI, 1991. MR 92e:46113 Zbl 0769.46036

[Ando and Haagerup 2013] H. Ando and U. Haagerup, "Ultraproducts of von Neumann algebras", preprint, 2013. arXiv 1212.5457

[Bratteli and Robinson 1997] O. Bratteli and D. W. Robinson, Operator algebras and quantum statistical mechanics, II: Equilibrium states, models in quantum statistical mechanics, 2nd ed., Springer, Berlin, 1997. MR 98e:82004 Zbl 0903.46066

[Connes and Størmer 1978] A. Connes and E. Størmer, "Homogeneity of the state space of factors of type III ${ }_{1}$, J. Functional Analysis 28:2 (1978), 187-196. MR 57 \#10435 Zbl 0408.46048

[Haagerup and Musat 2011] U. Haagerup and M. Musat, "Factorization and dilation problems for completely positive maps on von Neumann algebras", Comm. Math. Phys. 303:2 (2011), 555-594. MR 2012e:46125 Zbl 1220.46044

[Kishimoto et al. 2003] A. Kishimoto, N. Ozawa, and S. Sakai, "Homogeneity of the pure state space of a separable $C^{*}$-algebra", Canad. Math. Bull. 46:3 (2003), 365-372. MR 2004f:46072 Zbl 1066.46052

[Takesaki 1974] M. Takesaki, "Faithful states on a $C^{*}$-algebra”, Pacific J. Math. 52 (1974), 605-610. MR 50 \#8096 Zbl 0295.46092

Received February 27, 2013. Revised May 30, 2013. 
YASUYUKI KAWAHIGASHI

GRADUATE SCHOOL OF MATHEMATICAL SCIENCES

THE UNIVERSITY OF TOKYO

КомавА, ТOKYO, 153-8914

JAPAN

and

KAVLI IPMU (WPI)

THE UNIVERSITY OF TOKYO

5-1-5 KASHIWANOHA, KASHIWA, 277-8583

JAPAN

yasuyuki@ms.u-tokyo.ac.jp

YOSHIKO OGATA

GraduATE SCHOOL OF MATHEMATICAL SCIENCES

THE UNIVERSITY OF TOKYO

KOMABA, TOKYO, 153-8914

JAPAN

yoshiko@ms.u-tokyo.ac.jp

ERLING STøRMER

DEPARTMENT OF MATHEMATICS

UNIVERSITY OF OSLO

P.O. BOX 1053

BLINDERN, NO-0316 OSLO

NORWAY

erlings@math.uio.no 


\title{
PACIFIC JOURNAL OF MATHEMATICS
}

\author{
msp.org/pjm
}

Founded in 1951 by E. F. Beckenbach (1906-1982) and F. Wolf (1904-1989)

\section{EDITORS}

V. S. Varadarajan (Managing Editor)

Department of Mathematics

University of California

Los Angeles, CA 90095-1555

pacific@math.ucla.edu

Paul Balmer

Department of Mathematics

University of California

Los Angeles, CA 90095-1555

balmer@math.ucla.edu

Daryl Cooper

Department of Mathematics

University of California

Santa Barbara, CA 93106-3080 cooper@math.ucsb.edu

Jiang-Hua $\mathrm{Lu}$

Department of Mathematics

Pokfulam Rd., Hong Kong jhlu@maths.hku.hk
The University of Hong Kong

Don Blasius

Department of Mathematics University of California

Los Angeles, CA 90095-1555

blasius@math.ucla.edu

Robert Finn

Department of Mathematics Stanford University

Stanford, CA 94305-2125

finn@math.stanford.edu

Sorin Popa

Department of Mathematics

University of California

Los Angeles, CA 90095-1555 popa@math.ucla.edu

Paul Yang

Department of Mathematics Princeton University

Princeton NJ 08544-1000

yang@math.princeton.edu

\section{PRODUCTION}

Silvio Levy, Scientific Editor, production@msp.org

\section{SUPPORTING INSTITUTIONS}

ACADEMIA SINICA, TAIPEI

CALIFORNIA INST. OF TECHNOLOGY

INST. DE MATEMÁTICA PURA E APLICADA

KEIO UNIVERSITY

MATH. SCIENCES RESEARCH INSTITUTE

NEW MEXICO STATE UNIV.

OREGON STATE UNIV.

\author{
STANFORD UNIVERSITY \\ UNIV. OF BRITISH COLUMBIA \\ UNIV. OF CALIFORNIA, BERKELEY \\ UNIV. OF CALIFORNIA, DAVIS \\ UNIV. OF CALIFORNIA, LOS ANGELES \\ UNIV. OF CALIFORNIA, RIVERSIDE \\ UNIV. OF CALIFORNIA, SAN DIEGO \\ UNIV. OF CALIF., SANTA BARBARA
}

\author{
Vyjayanthi Chari \\ Department of Mathematics \\ University of California \\ Riverside, CA 92521-0135 \\ chari@math.ucr.edu \\ Kefeng Liu \\ Department of Mathematics \\ University of California \\ Los Angeles, CA 90095-1555 \\ liu@math.ucla.edu \\ Jie Qing \\ Department of Mathematics \\ University of California \\ Santa Cruz, CA 95064 \\ qing@cats.ucsc.edu
}

These supporting institutions contribute to the cost of publication of this Journal, but they are not owners or publishers and have no responsibility for its contents or policies.

See inside back cover or msp.org/pjm for submission instructions.

The subscription price for 2014 is US \$410/year for the electronic version, and \$535/year for print and electronic.

Subscriptions, requests for back issues and changes of subscribers address should be sent to Pacific Journal of Mathematics, P.O. Box 4163, Berkeley, CA 94704-0163, U.S.A. The Pacific Journal of Mathematics is indexed by Mathematical Reviews, Zentralblatt MATH, PASCAL CNRS Index, Referativnyi Zhurnal, Current Mathematical Publications and Web of Knowledge (Science Citation Index).

The Pacific Journal of Mathematics (ISSN 0030-8730) at the University of California, c/o Department of Mathematics, 798 Evans Hall \#3840, Berkeley, CA 94720-3840, is published twelve times a year. Periodical rate postage paid at Berkeley, CA 94704, and additional mailing offices. POSTMASTER: send address changes to Pacific Journal of Mathematics, P.O. Box 4163, Berkeley, CA 94704-0163.

PJM peer review and production are managed by EditFLOW ${ }^{\circledR}$ from Mathematical Sciences Publishers.

\section{PUBLISHED BY}

mathematical sciences publishers

nonprofit scientific publishing

http://msp.org/

(C) 2014 Mathematical Sciences Publishers 


\section{PACIFIC JOURNAL OF MATHEMATICS}

Volume $267 \quad$ No. $1 \quad$ January 2014

Numerical study of unbounded capillary surfaces

YASUNORI AOKI and HANS DE STERCK

Dual $R$-groups of the inner forms of $\operatorname{SL}(N)$

KUOK FAI CHAO and WEN-WEI LI

Automorphisms and quotients of quaternionic fake quadrics

AMIR DŽAmBIĆ and XAVIER ROULLEAU

Distance of bridge surfaces for links with essential meridional spheres 121

YEONHEE JANG

Normal states of type III factors

YASUYUKI KAWAHIGASHI, YOSHIKO OGATA and ERLING

STØRMER

Eigenvalues and entropies under the harmonic-Ricci flow

YI LI

Quantum extremal loop weight modules and monomial crystals

MATHIEU MANSUY

Lefschetz fibrations with small slope

NAOYUKI MONDEN 